\title{
The influence of complex drug cocarnit on the nerve conduction velocity in nerve tibialis of rats with diabetic polyneuropathy.
}

\author{
Nozdrenko DN*, Berehovyi SM, Nikitina NS, Stepanova LI, Beregova TV, Ostapchenko LI \\ ESC "Institute of Biology and Medicine", Taras Shevchenko National University of Kyiv, Kyiv, Ukraine
}

\begin{abstract}
We have analysed the influence of complex drug cocarnit ( $20 \mathrm{mg}$ nicotinamide, $50 \mathrm{mg}$ cocarboxylase, 0.5 mg cyanocobalamin, $10 \mathrm{mg}$ disodium adenosine triphosphate trihydrate) on the nerve conduction velocity in nervus tibialis of rats with diabetic polyneuropathy. Our data shows that diabetic polyneuropathy leads to dysfunction of signal conduction to the effector. Administration of complex drug cocarnit showed linear dose-dependent therapeutic effect on pathology. After 9 d course administration in rats with diabetic polyneuropathy Cocarnit led to almost complete time parameters renewal of stimulation impulses but only with the presence of long relaxation periods between the contractions. Cocarnit has great prospects as a remedy for diabetic polyneuropathy therapy.
\end{abstract}

Keywords: Skeletal muscle, Cocarnit, Diabetes, Polyneuropathy, Nicotinamide, Cocarboxylase, Cyanocobalamin.

Accepted on October 18, 2018

\section{Introduction}

Diabetic polyneuropathy is a frequent and progressing illness that worsens life quality greatly and followed by pronounced disability level. Polyneuropathy appears due to the dystrophy and degeneration changes in somatic and PNS (Peripheral nervous system) neurons. Distal symmetric sensorimotor polyneuropathy is observed much more frequently than central neuropathy under diabetes conditions [1]. As a basis of pathology lies progressive demyelination nerve fibers, segmental demyelination and axonal degeneration, as a result, nerve impulse conduction deceleration that changes all the chain of muscle activity control. Degeneration of distal sensory axons is also a prominent sign of diabetic peripheral neuropathy that influences on nerve conduction velocity as good as on the muscle answer pattern [2]. Progressive loss of neurons DRG (Dorsal Root Ganglia) under diabetic polyneuropathy which relates to neurotrophic rejection transforms the pattern of motoneuron pools from CNS (Central Nervous System). That fact makes accurate correction of muscle activity positioning almost impossible [3].

Contemporary pathogenetic therapy is directed to the main pathophysiological processes for preventing from nerve fibers damaging and deceleration of diabetic polyneuropathy progress. Nowadays we can see that therapy with the usage of group B vitamins and relevant derivatives that influences directly on the damaged tissues is rather popular [4].

Nicotinamide is a component of $\mathrm{CO}$ dehydrogenase and influences on the reduction-oxidation processes in the cell. The influence of nicotinamide on the reduction-oxidation processes in the streptozotocin-induced experimental diabetes leads to prominent blood glucose level reduction after administration of this vitamin. It is also known that nicotinamide increases the SOD (superoxide dismutase) activity in animals with induced diabetes [5]. Nicotinamide improves general physiological state in patients with diabetes [6]. It is also known that administration of nicotinamide reduces inflammation and oxidative stress of retinopathy because of long-lasting negative consequences of hyperglycemia [7].

Thiamine plays an importation role in carbohydrate, protein and lipid metabolism, synaptic nerve conduction etc. It also protects cell membrane from toxic influence of peroxide oxidation products. The lack of thiamine under diabetic condition leads to the nerve conduction disturbance and loss of muscle coordination [8]. Thiamine is also a modulator of neuro-muscular conduction, and neurotransmission can be broken by thiamine antagonist-pyrithiamine. Electrophysiological researches showed prominent therapeutic effect of thiamine on the diabetic neuropathy development. Thiamine administration influenced on the nerve restoring changes of motor conduction and distal latency velocity in patients with diabetic neuropathy [9]. Thiamine therapy administration (dose $75 \mathrm{mg}$ per day) prominently reduces daily dose demand of insulin and improves consequences of macrocytic anemia in patients under diabetic conditions. It is worth mentioning that discontinue of thiamine therapy leads to the insulin demand growth and macrocytic anemia relapse [10].

Nevertheless, therapeutic doses of thiamine exceed normal doses in 100-1000 times, and as thiamine is water-soluble vitamin, it is almost impossible to gain high thiamine concentrations directly in the cell. That is the reason why we 
think it is better to use thiamine therapy in combination with the drug that discourage progressive reduction of the vitamin in the cell under diabetic conditions.

Cocarboxylasum is a non-protein coenzyme that improves activity of many enzymes and tissue metabolism. Many enzymes that catalyses carboxylation and decarboxylation keto acids, pyretic acid, promotes to form acetyl-coenzyme A need coenzyme cocarboxylase for their normal functioning, and this determines its role in carbohydrate metabolism. Involvement in pentose cycle promotes nucleic acids, peptides and lipids synthesis, improves nerve tissue trophism, take part in CNS function renewal.

Many researches showed that therapeutic administration of cocarboxylasum prevents the decrease of thiamine concentration in the patients' blood that suffers from the kidney failure. Prominent rise of the thiamine level in the blood plasma can be seen even after several days of administration of cocarboxylasum, that support the curing of potential thiamine deficit and metabolic disorders during the development of this pathology [11].

Cyanocobalamin (Vitamin B12) is contained in cocarnit. This vitamin is transferred in organism into active formadenosinecobalamin which has high biological activity, refines carbohydrate and lipid metabolism, and improves nerve tissue regeneration. Low level of $\mathrm{B} 12$ is a common problem for patients with diabetes that enhances following progressive dysfunctions of muscular control [12]. At the same time usage B12 as a therapy of diabetic peripheral neuropathy showed prominent positive dynamics [13].

Many studies show that many therapeutic agents with positive dynamics in diabetic polyneuropathy treatment are followed by B12 deficit. That fact significantly declines the beneficial effect of a medicine. It is also worth mentioning that insulindependent patients with B12 metabolism changes under diabetic conditions suffer from high concentration of Cyanocobalamin in the erythrocytes and high velocity of B12 urine excretion [14].

In this research we explore the influence of complex drug Cocarnit on the nerve impulse conduction dynamics in nervus tibialis (SI and SII) that innervates musculus gastrocnemius under diabetic polyneuropathy condition in rats. This drug contains efficient complex of metabolic substances and vitamins: nicotinamide-20 $\mathrm{mg}$, cocarboxylasum-50 mg, cyanocobalamin- $0.5 \mathrm{mg}$, dissodium adenosine triphosphate trihydrate-10 $\mathrm{mg}$ [15]. The choice of drug is based on the point that each component develops therapeutic effect on different physiological levels.

Thus, considering the pathogenesis of diabetic polyneuropathy, investigation healing effect of the drugs based on group B vitamins became main aim of this investigation.

\section{Materials and Methods}

\section{Modeling of diabetic neuropathy}

Modeling of diabetic neuropathy was performed on 100 rats (50 males and 50 females). Rats were induced diabetes type I as described by Islam et al. [16]. Experimental mature rats with weight $180-200 \mathrm{~g}$ were administrated by streptozotocin (Streptozotocin, «Sigma», USA) interperitonealy in a dose 65 $\mathrm{mg} / \mathrm{kg}$. To confirm the presence of diabetes glucose level check was performed before the pathology, on 14, $28 \mathrm{~d}$ of experiment and glucose tolerance test was performed at $30 \mathrm{~d}$. Polyneuropathy was detected at the $28 \mathrm{~d}$ which progressed dramatically. The presence of polyneuropathy was also confirmed by using analgesiometer.

\section{Biomechanical analysis}

Animals in deep narcosis (ketamine (20 mg/kg) "Pfizer", USA) were operated (tracheotomy was performed) and connected to artificial lung ventilation machine. At the area of popliteal fossa musculus gastrocnemius was isolated and cut down proximally. Then musculus gastrocnemius was connected to the strain gage force sensor [17]. The nerve that innervates $\mathrm{m}$. gastrocnemius was fixed on the bipolar platinum electrode for further electrostimulations. Parameters of the stimulation signal were programmed automatically [18]. During operation and experiment the heart rate and ECG amplitude were monitored.

Distinguishing of motoneuron nerve conduction changes was performed by measuring the time differences of the beginning of muscle answer. As a test stimulation pools with 2 periods of relaxation (10 $\mathrm{s}$ and $3 \mathrm{~min}$ ) were used. It gave us an opportunity to analyse connection of diabetic polyneuropathy with the fatigue processes development.

Drug cocarnit (World medicine) diluted in $0.5 \%$ solution of lidocaine was administrated intramuscularly in dose $1 \mathrm{mg} / \mathrm{kg}$ during 3, 6 and $9 \mathrm{~d}$. The duration of administration of Cocarnit was picked up experimentally. Provided data reflects dose loading that have the most prominent therapeutic effect. Worth mentioning, that prolonged therapy more than $9 \mathrm{~d}$ displays no improvement in the present experiment.

Data analysis including statistical treatment and graph plotting was performed using Origin 8.0 software ("Origin Lab Corp.", USA). P values less than 0.05 were used to indicate statistically significant differences in the mean values of parameters measured. The data followed Gaussian distributions and had identical standard deviations (s.d.). Values are given as the means \pm s.e.m; $\mathrm{n}$ represents the number of experiments. To determine the statistical significance of differences between the group means, a Student t-test was used.

\section{Results and Discussion}

As marker of glucose metabolism disturbance in the body serves hyperglycemia, so after the introduction of streptozotocin we examined blood glucose levels every $7 \mathrm{~d}$. In 
the experiment we took only those animals, which developed hyperglycemia. As is known from the literature after the introduction of streptozotocin diabetes develops on the $30^{\text {th }} \mathrm{d}$. Therefore, $30 \mathrm{~d}$ after the start of the simulation of diabetes, the animals conducted a glucose tolerance test. We showed that the level of glucose in the rat blood was much higher than the control group. Moreover, $2 \mathrm{~h}$ after the start of the test, this value is not decreased even after $2 \mathrm{~h}$. This is a direct indication of the development of diabetes in the experimental rats (Figure 1).

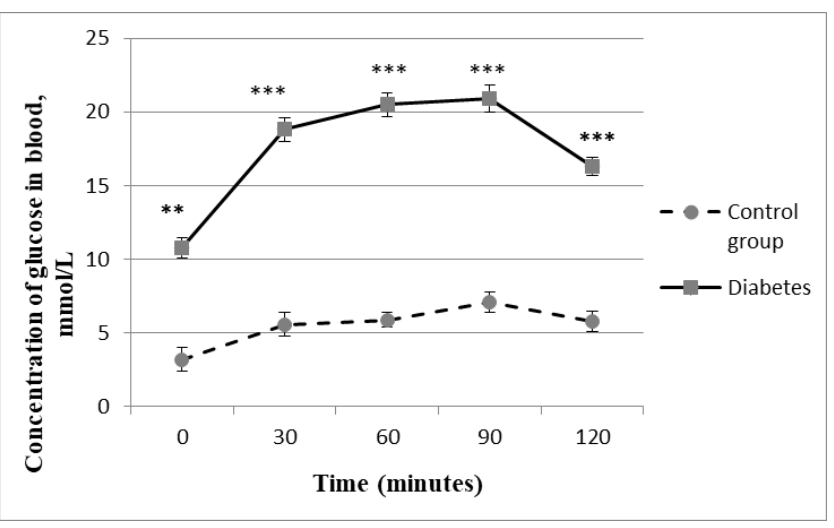

Figure 1. The level of glucose in the blood of rats after 30, 60, 90 and 120 min after the introduction of glucose, mmol/l. ${ }^{* * *} P<0.001$.

Motor unit-motor-neuron and population of muscle fibers which it innervates represents one of the most important functional elements of the motor control system. Motorneurons are discharged continuously in response to steady super threshold activation of their exciting entrances by difficult stimulation pools. Dysfunction of this system leads to serious violations. Axons of motor neurons are susceptible to diabetes, and their damage can cause complications in maintaining the balance of movements. In experiments on mice with diabetes, there are many abnormalities that indicate the degeneration of axons, besides, the abnormal axon morphology leads to a significant variability of their thickness, which is reflected on the speed of the nerve transmission [19]. The number of small motor neurons that innervate muscles gastrocnemius significantly decreased after the induction of streptozotocin diabetes in several weeks [20]. After $10 \mathrm{w}$, patients with diabetes almost did not have small motor neurons, and the size distribution became unimodal. It was found that there is a significant reduction in the absolute number of motor neurons innervating $\mathrm{m}$. gastrocnemius in diabetic rats, and this decrease is occurring first in the smaller motor neurons [20].

Temporary parameters of carrying out stimulation pools on an axon do not remain constants, either when the intensity of stimulation changes or when the relaxation time changes. The study of the changes in the temporal delays in impulse conduction at the increase of impulses quantity allowed evaluating the level of pathological processes in the neuromuscular preparation with long, static and slow dynamic reactions of the muscular system. Figure 2 shows the mechanograms of change in response time at the beginning of the power answer of muscles gastrocnemius induced by consecutive stimulation pools with relaxation time $3 \mathrm{~min}$ in rats with diabetic neuropathy at administration of cocarnit for 3,6 and $9 \mathrm{~d}$.

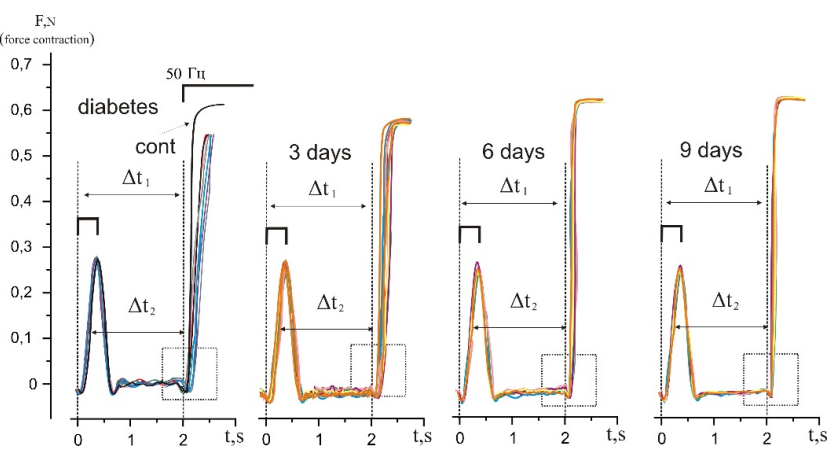

Figure 2. Mechanograms of muscle gastrocnemius start time of response caused by irritation consecutive stimulations pools with a frequency of $50 \mathrm{~Hz}$ in rats with diabetes and administration of cocarnit during 3, 6 and $9 \mathrm{~d}$. Relaxation time is $3 \mathrm{~min}$. Cont-control, $\Delta t 1$-time between two consecutive pools of stimulation, $\Delta t 2$-time of the beginning of the muscular response to the applied stimulation. 3 , 6 and 9 d-mechanograms at administered cocarnit for 3, 6 and $9 d$.

Calculation of time between consecutive mitotic answers $(\Delta \mathrm{t} 2)$ caused by stimulation (Figure 2 ) with the known time $(\Delta \mathrm{t} 1)$ between pools and long relaxation ( $3 \mathrm{~min}$ ), made it possible to evaluate the presence of neuropathic dysfunctions which are not associated with the development of fatigue processes and to correct algorithm of further therapeutic influence. The analysis of change of time of the power answer of muscles gastrocnemius with diabetic polyneuropathy (Figures 2 and 3) showed that time of beginning of the power answer increased from $119.34 \%$ at the first stimulation pool to $132.2 \%$ at the tenth (the control data which were not changing the parameters throughout all experiment were accepted to $100 \%$ ). Thus, it is possible to note the presence of expressed fatigue processes of neuromuscular preparation, even at the long relaxation periods. It should be noted that time of the beginning of the force response with direct stimulation of the muscle did not change, which indicates the absence of myopathy pathological manifestations under these conditions.

The use of cocarnit, revealed a linear dose-dependent therapeutic effect on this pathology (Figures 2 and 3). $3 \mathrm{~d}$ administration of the drug reduced temporary delays in activation of muscular activity from $112.56 \%$ at the first reduction to $125.76 \%$ at the tenth. Administration of the drug for $6 \mathrm{~d}$ increased this effect to $107 \%$ at the first stimulation to $115 \%$ at the tenth (Figures 2 and 3). Nine-day therapy brought the studied parameters almost to control values and made $100 \%$ and $104.66 \%$ at the first and tenth reduction respectively. From data obtained it can be concluded that the cocarnit preparation with $9 \mathrm{~d}$ therapy of rats with the diabetic neuropathy induced by streptozotocin leads to almost complete recovery of temporary parameters of carrying out stimulation impulses on nervous tibialis to the innervating muscles gastrocnemius at application of the long periods of relaxation between reductions. 


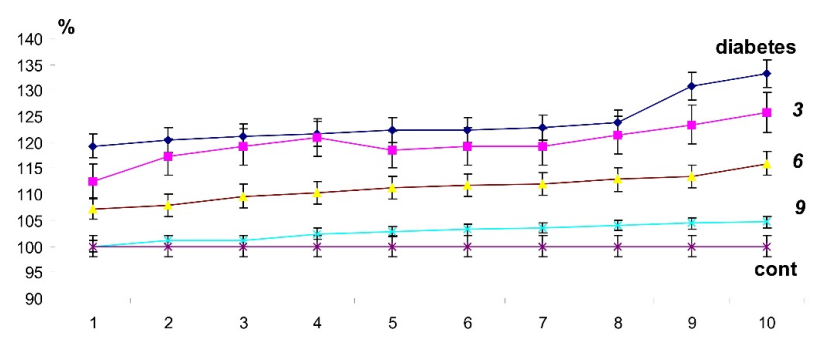

Figure 3. Change in the start time of power answers of musculus gastrocnemius with diabetes is caused in rats, caused by 10 consecutive pools of stimulation by electrostimulation at $50 \mathrm{~Hz}$. Relaxation time is $3 \mathrm{~min}$. Data are presented as a percentage of the control values taken for 100\% 1-10-consecutive pools irritation, diabetes-diabetic rat, Cont-control rat, 3, 6 and 9-irritation at introduction to cocarnit current of 3, 6 and 9 d respectively.

In the detailed analysis of the pathological processes development connected with the polyneuropathy, it is necessary to use non-refractory stimulation pools that cause prolonged trans synaptic activation of motoneurons. At the same time there is noticeable characteristic adaptation decrease in time of impulse conductance on nervous tissue induced by development of fatigue processes. Change of this indicator is characteristic marker of the presence of pathological processes in the neuromuscular drug when applying stimulation signals close to physiological parameters.

The analysis of muscles gastrocnemius changes in power answer caused by stimulation pools with a frequency of $50 \mathrm{~Hz}$ in rats with diabetic polyneuropathy at application 10 second relaxation between them showed that time of power answer at beginning increased from $124.12 \%$ at the first reduction to $142.27 \%$ at tenth (Figures 4 and 5). It should be noticed, that in this case the time of muscle force response at beginning with direct stimulation also did not change. From the obtained data we can conclude about the presence of the neuropathic changes connected with the inability to implement even 10 consecutive stimulation pools without significant physiological disturbances of neurotropic origin.

The three-day therapy with cocarnit reduced these indicators by $119.97 \%$ at the first and for $132.55 \%$ at the tenth reduction which is not a very significant improvement in the pathological process under study in these conditions (Figures 4 and 5). Increase in dose loading drug up to $6 \mathrm{~d}$ of continuous introduction, increased time of carrying out a nervous impulse almost by $50 \%$ in comparison with diabetic animals and made $105.88 \%$ and $114.85 \%$ at the first and tenth reduction respectively. Application $9 \mathrm{~d}$ therapy by Cocarnit led to increase in the described temporary delays up to $102.33 \%$ and $105.91 \%$ at the first and tenth reduction respectively but did not lead to the achievement of the control level. Thus, the obtained data show that the polyneuropathy caused by diabetes leads to significant dysfunctions in the transmission of the stimulatory signal to the effector. When the parameters of signal stimulation approach the physiological level, the dysfunction of neuromuscular activity increases to a level capable of disturbing the overall dynamics of the contractile process.
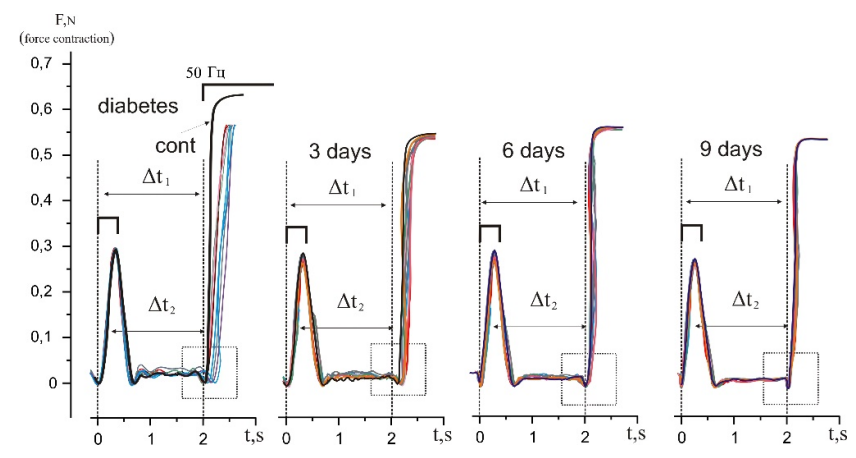

Figure 4. Mechanograms of muscle gastrocnemius beginning time response caused by irritation consecutive stimulations pools with a frequency of $50 \mathrm{~Hz}$ in rats with diabetes and administration of cocarnit during 3, 6 and $9 \mathrm{~d}$. Relaxation time is $10 \mathrm{~s}$. Cont-control, $\Delta t 1$-time between two consecutive pools of stimulation, $\Delta t 2$-time of the beginning of the muscular response to the applied stimulation. 3 , 6 and 9 d-mechanograms at administered cocarnit for 3, 6 and $9 d$.

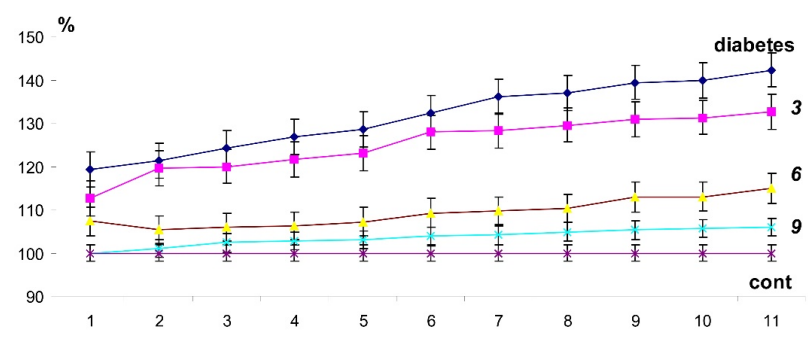

Figure 5. Change in the time of the beginning power answers of musculus gastrocnemius with diabetes is caused in rats, caused by 10 consecutive pools of stimulation by electrostimulation at $50 \mathrm{~Hz}$. Relaxation time is $10 \mathrm{~s}$. Data are presented as a percentage of the control values taken for 100\% 1-10-consecutive pools irritation, diabetes -diabetic rat, cont-control rat, 3, 6 and 9-irritation at introduction to cocarnit current of 3, 6 and 9 d respectively.

Today, the cause of the development of peripheral neuropathy in patients with diabetes is considered not only as hyperglycemia. The role of other non-glycemic factors such as insulin-like in type 1 diabetes and insulin-resistant in type 2 diabetes is considered. Both cases are essentially the only mechanism violation of insulin signaling [21]. Also, the role of IGFs (insulin-like growth factors) and C-peptides (a fragment of proinsulin molecule) as an intermediary in the pathogenesis of neuropathy is discussed [21]. Diabetes triggers also the cascade of other metabolic dysfunctions in human body which can indirectly influence the kind of nerve transmission. So, at diabetes of the second type considerable deterioration in the central control of motor reactions and osteogenic dysfunction is observed [22]. Thus, development of this pathology is multifactorial and despite long-term efforts, remains badly clear. Therefore, it is difficult to identify the main factors that influence the reduction of the time of nerve conduction in the development of diabetes. And the more difficult it becomes to decide the question of finding adequate therapeutic drugs capable of bringing neuronal violations to physiological norms. 
However, the ability of cocarnit to reduce the difference in nerve conduction between the pathological and control status (Figures 2 and 4) gives great prospects for its application.

Studies in animals with 8-month-old streptozotocin induced diabetes indicate that motor neurons withdraw their terminals from distal innervation. That is, there are progressive features of distal loss of axons, but without loss of reburial (death of the body of the neuron), indicating a distal retraction of axons. In this case, although the size and size of the motor neuron are preserved, the rate of conduction of potentials of action on axons decreases with each successive reduction and there was a compensatory increase in the potential of action in response to single irritation $[23,24]$. This process may correspond to the increase described above for pathological myotonic dysfunctions with an increase in the frequency of contraction (a decrease in the relaxation time), and a reduction in the therapeutic effect of Cocarnit on these, almost non-inverse pathological processes (Figures 4 and 5).

It should be noted that studies in diabetic rats indicated no significant electrophysiological changes in the functioning of $\alpha$-motor neurons at the lumbar level [25]. However, significant changes occurred in trace hyperpolarization, namely, decreased after hyperpolarization half-decay (AHP1) and after hyperpolarization amplitude (AHPamp) [25]. What, according to researchers, is the first sign of the inherent pathological changes in the function of $\alpha$ - motor neurons for diabetes. It should also be noted that, by quantitative electromyography studies, it was found that in diabetic polyneuropathy, the number of functioning motor units decreases by $60 \%$ compared with control. However, the authors emphasize that it is still too early to associate the number of non-functional motor units with the stage of polyneuropathy in each case [26]. But the decrease in the number of motor units and as the increase in the number of active myocytes that are innervated with one axon will surely be associated with the inability of the nerves to transfer the action potentials to the active muscle adequately, so that to begin the process of reducing the whole muscle (fixed by us in the experiment) moto neurons need to activate most of functioning myocytes. Our data show that complex long-term therapy with nicotinamide, thiamine and cyanocobalamin is likely to reduce the number of damaged motor neurons in the muscles gastrocnemius units in the development of diabetic neuropathy, but the mechanisms of this effect require further investigation.

Thus, a significant variety of induced pathological changes in diabetic neuropathy suggests that they are heterogeneous. To understand the dynamics of the development of diabetic polyneuropathy, it is necessary to simultaneously analyse two mechanisms. The first one, which suggests that hyperglycemia induces metabolic disorders that directly affect the cells of Schwann (or myelin), intercept rangier or axons. The second is that hyperglycemia and metabolic disorders affect the structure and function of connective tissues that cover the myelin shells of the nerve fibers of the spinal cord, which then induce the development of neuropathy by altering the blood-nervous tissue barrier, causing hypoxia or ischemia or other unknown mechanism [27].

There is also increasing evidence that diabetic neuropathy induces nerve damage by the inflammatory (immune) vasculitis, which causes complete nerve fiber dysfunction [27]. But in any case, diabetic neuropathy leads to the inhibition of the dynamic parameters of the contraction of skeletal muscles due to the time delay of realizing muscle efferent irritation. Our studies have shown that these delays increase with increasing time of active muscle work. This makes it impossible to adequately realize the motor neuronal bullets by the muscular system, which will have significant consequences for precise movements [28]. The long-term therapeutic introduction of the drug Cocarnit can substantially improve the described dysfunction and in some cases to bring the mechanokinetic parameters of the reduced muscle to the levels of the physiological norm. Thus, the drug cocarnit has significant prospects as a means of correcting diabetic polyneuropathy and has a high practical value.

The received results indicate complexity to combined therapy with participation of the vitamins $B$ in optimum model of diabetic polyneuropathy, namely for the adequate description of all physiological processes blocking full range of movements. The biomechanical analysis including detailed calculation of kinetic and dynamic characteristics in neuromuscular medicine can help with determination of the level of pathological processes in nervous system caused by diabetes and to define factors in optimization of therapy of these pathologies.

\section{References}

1. Sohn EH, Song KS, Lee JY, Lee AY. Comparison of somatic and sudomotor nerve fibers in type 2 diabetes mellitus. J Clin Neurol 2017; 13: 366-370.

2. Francis GJ, Martinez JA, Liu WQ, Zochodne DW, Hanson LR, Frey WH, Toth C. Motor end plate innervation loss in diabetes and the role of insulin. $\mathrm{J}$ Neuropathol Exp Neurol 2011; 70: 323-339.

3. Kamiya H, Zhang W, Sima AA. Degeneration of the Golgi and neuronal loss in dorsal root ganglia in diabetic BioBreeding/Worcester rats. Diabetologia 2006; 49: 2763-2774.

4. Várkonyi T, Korei A, Putz Z, Martos T, Keresztes K. Advances in the management of diabetic neuropathy. Minerva Medica 2017; 108: 419-437.

5. Opoka-Winiarska V, Tuszkiewicz-Misztal E. Evaluation of nicotinamide influence on selected parameters of oxidative/antioxidative balance in animals with streptozotocin induced diabetes. Diabetologia Polska 1999; 6: 16-23.

6. Kodentsova VM, Vrzhesinskaia OA, Sokolnikov AA. Effectiveness of the use of vitamin-enriched food premixes in the nutrition of children with insulindependent diabetes. Voprosy Pitaniia 1993; 5: 40-45. 
7. Karbasforooshan H, Karimi G. The role of SIRT1 in diabetic retinopathy. Biomed Pharmacother 2018; 97: 190-194.

8. Tubas F, Akyildiz B, Ozcan A, Kurtolu S, Akin L, Baykan A. Thiamine-responsive supraventricular tachycardia in a patient with diabetes mellitus. Cocuk Sagligi ve Hastaliklari Dergisi 2014; 57: 262-264.

9. Cvijanovic M, Simic S, Kopitovic A, Raicevic R. Neurophysiological evaluation of short-term outcome of pharmacological treatment of diabetic neuropathy. Vojnosanitetski Pregled 2017; 74: 722-727.

10. Akinci A, Tetzic T, Erturk G, Tarim O, Dalva K. Thiamine-responsive megaloblastic anemia with diabetes mellitus and sensorineural deafness. Pediatr Int 1993; 35: 262-266.

11. Pietrzak I, Czarnecki R, Baczyk K, Mlynarczyk M, Kaczmarek M. The effect of cocarboxylase treatment on erythrocyte transketolase and blood thiamine in patients with end stage renal disease undergoing maintenance hemodialysis. Przegla d lekarski 2000; 57: 369-373.

12. Fogelman Y, Kitai E, Blumberg G, Rapoport M, Carmeli E. Vitamin B12 screening in metformin-treated diabetics in primary care: were elderly patients less likely to be tested? Aging Clin Exp Res 2017; 29: 135-139.

13. Roy RP, Ghosh K, Ghosh M, Acharyya A, Bhattacharya A, Pal M, Chakraborty S, Sengupta N. Study of vitamin B12 deficiency and peripheral neuropathy in metformintreated early type 2 diabetes mellitus. Indian J Endocrinol Metab 2016; 20: 631-637.

14. Kodentsova VM, Vrzhesinskaia OA, Sokolnikov AA, Kharitonchik LA, Spirichev VB. Metabolism of B group vitamins in patients with insulin-dependent and noninsulin dependent forms of diabetes mellitus. Voprosy Meditsinskoj Khimii 1993; 39: 26-29.

15. Cocarnit-World Medicine 2018

16. Islam MS, Wilson RD. Experimentally induced rodent models of type 2 diabetes. Anim Model Diab Res 2012; 933: 161-174.

17. Nozdrenko DM, Abramchuk OM, Soroca VM, Miroshnichenko NS. Aluminum chloride effect on $\mathrm{Ca} 2+$, $\mathrm{Mg} 2+-$ ATPase activity and dynamic parameters of skeletal muscle contraction. Ukrainian Biochem J 2015; 87: 38-45.

18. Khoma OM, Zavodovskya DA, Nozdrenko DN, Dolhopolov OV, Miroshnychenko MS, Motuziuk OP. Dynamics of ischemic skeletal soleus muscle contraction in rats. Fiziol Zh 2014; 60: 34-40.
19. Muller KA, Ryals JM, Feldman EL, Wright DE. Abnormal muscle spindle innervation and large-fiber neuropathy in diabetic mice. Diabetes 2008; 57: 1693-1701.

20. Muramatsu K, Niwa M, Nagai M, Kamimura T, Sasaki S, Ishiguro T. The size of motoneurons of the gastrocnemius muscle in rats with diabetes. Neurosci Lett 2012; 531: 109-113.

21. Dobretsov M, Romanovsky D, Stimers JR. Early diabetic neuropathy: triggers and mechanisms. World J Gastroenterol 2007; 13: 175-191.

22. Wallner C, Jaurich H, Wagner JM, Becerikli M, Harati K, Dadras M, Lehnhardt M, Behr B. Inhibition of GDF8 (Myostatin) accelerates bone regeneration in diabetes mellitus type 2. Sci Rep 2017; 7: 9878.

23. Ramji N, Toth C, Kennedy J, Zochodne DW. Does diabetes mellitus target motor neurons? Neurobiol Dis 2007; 26: 301-311.

24. Nozdrenko DN, Bogutska KI. About molecular mechanisms of fiber muscle contraction at transition to new equilibrium state: analysis of experimental data using three-componential electrical stimulating signal. Biopolymers Cell 2005; 21: 283-286.

25. Macdonell CW, Chopek JW, Gardiner KR, Gardiner PF. Motoneurons maintain biophysical heterogeneity in obesity and diabetes in Zucker rats. J Neurophysiol 2017; 118: 2318-2327.

26. Allen MD, Choi IH, Kimpinski K, Doherty TJ, Rice CL. Motor unit loss and weakness in association with diabetic neuropathy in humans. Muscle Nerve 2013; 48: 298-300.

27. Dyck PJ, Giannini C. Pathologic alterations in the diabetic neuropathies of humans: a review. J Neuropathol Exp Neurol 1996; 55: 1181-1193.

28. Nozdrenko DM, Miroshnychenko MS, Soroca VM, Korchinska LV, Zavodovskiy DO. The effect of chlorpyrifos upon ATPase activity of sarcoplasmic reticulum and biomechanics of skeletal muscle contraction. Ukrainian Biochem J 2016; 88: 82-88.

\section{*Correspondence to}

Nozdrenko DN

ESC "Institute of Biology and Medicine"

Taras Shevchenko National University of Kyiv

Ukraine 